\title{
Effects of an Axial Magnetic Field on Vortex Breakdown and Fluid Layer
}

\author{
B. Mahfoud ${ }^{\dagger}$ \\ Department of Mechanical, University of UAMO-Bouira, 10000, Algeria \\ $\dagger$ Corresponding Author Email: mahfoud.mah@gmail.com
}

(Received January 15, 2021; accepted June 1, 2021)

\begin{abstract}
The effects of an axial magnetic field on both the vortex breakdown process and fluid layers development in a cylindrical container filled with a conducting viscous fluid are numerically analyzed by using the Generalized Integral Transform Technique (GITT) with a stream function-only formulation. A temperature gradient is imposed in the axial direction on the swirling flow which is advanced by the rotation of the bottom disk under the stabilizing effect of the external magnetic field. Flows are studied for a range of parameters: the Richardson number, $\mathrm{Ri}, 0 \leq \mathrm{Ri} \leq 2.0$; and three values of the Prandtl number are investigated, $\operatorname{Pr}=0.025$ (liquid Mercury), 0.032 ( $\mathrm{PbLi} 17$ alloy), and 0.065 (the molten lithium). Three combinations of aspect ratios (H/R) and Reynolds numbers are compared: (case $\mathrm{A}$ : $\mathrm{Re}=1500, \mathrm{H} / \mathrm{R}=1.5$ ); (case $\mathrm{B}$ : $\mathrm{Re}=1855, \mathrm{H} / \mathrm{R}=2.0$ ) and (case $\mathrm{C}$ : $\mathrm{Re}=2400$, $\mathrm{H} / \mathrm{R}=2.5$ ). The results reveal that the increase in the values of Hartmann number, Ha suppresses the vortex breakdown in the isothermal case and reduces the number of fluid layers in the layering case. The stability diagram $(\mathrm{Ha}$ - $-\mathrm{Ri}$ ) corresponding to the transition from the multiple fluid layers zone to the one fluid layer zone for increasing Prandtl number is obtained.
\end{abstract}

Keywords: Fluid layers; Integral transforms; Magnetic field; Swirling flow; Vortex breakdown.

\section{NOMENCLATURE}

\begin{tabular}{|c|c|c|c|}
\hline $\mathrm{B}_{0}$ & $\begin{array}{l}\text { magnitude of the external magnetic } \\
\text { field, Tesla }\end{array}$ & Greek Letters & \\
\hline$F_{L}$ & dimensionless Lorentz force & $\alpha$ & fluid thermal diffusivity \\
\hline$g$ & gravity & $\beta$ & $\begin{array}{l}\text { coefficient of thermal expansion of } \\
\text { the fluid }\end{array}$ \\
\hline$H$ & height of the cylinder , $\mathrm{m}$ & $\beta_{\mathrm{i}}$ & $\begin{array}{l}\text { eigenvalues for the temperature } \\
\text { expansion }\end{array}$ \\
\hline$H a$ & Hartmann number & $\gamma \mathrm{i}$ & $\begin{array}{l}\text { eigenvalues for the streamfunction } \\
\text { expansion }\end{array}$ \\
\hline $\mathrm{J}$ & dimensionless current density & $\Gamma_{\mathrm{i}}$ & $\begin{array}{l}\text { eigenfunctions for the temperature } \\
\text { expansion }\end{array}$ \\
\hline $\mathrm{L}_{\mathrm{i}}$ & $\begin{array}{l}\text { normalization integral for the } \\
\text { streamfunction }\end{array}$ & $\Delta \mathrm{T}$ & $\begin{array}{l}\text { temperature difference between the } \\
\text { top and the bottom }\end{array}$ \\
\hline $\mathrm{Mi}_{\mathrm{i}}$ & $\begin{array}{l}\text { normalization integral for the } \\
\text { azimuthal velocity component }\end{array}$ & $\Theta$ & dimensionless temperature \\
\hline $\mathrm{N}$ & truncation order & $\lambda \mathrm{i}$ & $\begin{array}{l}\text { eigenvalues for the azimuthal } \\
\text { velocity component expansion }\end{array}$ \\
\hline $\mathrm{N}_{\mathrm{i}}$ & $\begin{array}{l}\text { normalization integral for the } \\
\text { temperature field }\end{array}$ & $\theta$ & angular coordinate \\
\hline$P$ & dimensionless pressure & $\mu_{\mathrm{i}}$ & $\begin{array}{l}\text { eigenvalues for the temperature } \\
\text { expansion }\end{array}$ \\
\hline $\operatorname{Pr}$ & Prandtl number & $v$ & kinematic viscosity \\
\hline$R$ & radius of the cylinder & $\lambda$ & thermal conductivity \\
\hline$r, \theta, z$ & dimensionless spatial coordinates & $\rho$ & fluid density \\
\hline$R e$ & Reynolds number & $\psi$ & hydrodynamic streamfunction \\
\hline$R e_{\mathrm{m}}$ & magnetic Reynolds number & $\psi^{\prime}$ & electric streamfunction \\
\hline
\end{tabular}




\begin{tabular}{|c|c|}
\hline$R i$ & Richardson number \\
\hline$T_{0}$ & reference temperature \\
\hline $\mathrm{v}_{r}, \mathrm{v}_{\theta}, \mathrm{v}_{z}$ & $\begin{array}{l}\text { dimensionless radial, azimuthal, axial } \\
\text { velocity components }\end{array}$ \\
\hline $\mathrm{V}$ & dimensionless velocity vector \\
\hline $\mathrm{X}_{\mathrm{i}}$ & $\begin{array}{l}\text { eigenfunctions for the streamfunction } \\
\text { expansion }\end{array}$ \\
\hline $\mathrm{Yi}_{\mathrm{i}}$ & $\begin{array}{l}\text { eigenfunctions for the azimuthal } \\
\text { velocity component expansion }\end{array}$ \\
\hline
\end{tabular}

\section{INTRODUCTION}

One of the methods researchers implemented to control or suppress the vortex breakdown is the use of magnetohydrodynamics. The use of the uniform magnetic field is known as a flow stabilizer. For example, in the crystal growth process, the magnetic field is used to control the flow in the melt, and consequently, a good crystal quality is obtained (Oreper et al. 1984). The continuous casting process also uses the magnetic field to control the flow of the steel and minimize defects in the final products (Davidson et al.2001). In the tokamak idea, an intense magnetic field is applied to retain the plasma far off the reactor walls (Mahfoud et al. 2020). In swirling flow in the cylinder container, an Ekman boundary layer which describes the force balance between the viscous and centrifugal forces forms near the rotating disk. If the amplitude of the magnetic field grows, the Hartmann layer represents the electromagnetic forces that replace the Ekman layer. The literature review on convection under an external magnetic field confirmed that it can be used for the control and stabilization of the fluid motion (Mahfoud and Bessaih 2012, 2016. Mahfoud et al. 2016, 2019). Other (Bendjaghlouli et al. 2019a, $2019 \mathrm{~b}$ ) have discussed the problems of the rotating fluid in containers with different geometry under an external magnetic field. The effect of the electrical conductivity of the walls on the fluid flow cannot be neglected, as was investigated in the studies of Kharicha et al. (2004, 2005). Some researchers have studied the possibility to use a magnetic field to control the behavior of the vortex breakdown and stabilize the flow (Yu et al. 2013; Dash and Singh 2019). Laouari et al. (2021) have recently studied the effects of the electrical conductivity of the wall on the vortex breakdown position and its disappearance in the different regimes. The results obtained be seen that the vortex breakdown is eliminated after the magnitude of the magnetic field surpasses a critical value. A stability diagram corresponding to the transition from the vortex zone to the no-vortex zone for insulating and electrically conducting walls was provided.

The present study uses the Generalized Integral Transform Technique (GITT), which is a hybrid method for solving linear or nonlinear diffusion and convection-diffusion problems. This method has been can be used for MHD fluid flow problems, as reviewed in various sources (Lima et al. 2013; Matt et al. 2017).

In this paper, GITT approach is used to identify critical Hartmann numbers, $\mathrm{Ha}_{\mathrm{cr}}$ (i.e, the most

\author{
$\sigma_{e} \quad$ electric conductivity \\ $\Phi$ dimensionless electric potential \\ $\Omega \quad$ angular velocity

\section{Subscripts and superscripts} \\ $\mathrm{i}, \mathrm{j}, \mathrm{k} \quad$ orders from eigenvalue problems \\ transformed quantities
}

efficient magnitude of the magnetic field) corresponding to removing both vortex breakdown and fluid layers. Therefore, we present the limits that correspond to the domain where layering does not occur.

\section{Flow CONFIGURATION AND MODEL}

\subsection{Model}

Electrically conducting fluid fills a cylindrical container of radius $\mathrm{R}$ and height $\mathrm{H}$ which the value of $H / R$ for the case considered is equal to 1.5, 2.0, and 2.5 , respectively. The steady swirling flow is supposed to be laminar, and incompressible. A temperature difference $(\Delta \mathrm{T})$ is axially imposed (the top disk is hotter than the bottom), whereas the sidewall is supposed to be thermally insulated. The entire system (fluid and solid walls) is subjected to an external axial magnetic field, $\boldsymbol{B}\left(B_{0} \mathbf{e}_{\mathrm{z}}\right)$ acting in the $z$-direction. The rotating motion is generated by the lower disk which rotates at a constant angular velocity $\Omega$, while the upper disk is fixed. The magnetic Reynolds number $R e_{m}=\mu_{0} \sigma \Omega R^{2} \ll 1$, that measure the rapport of induction to magnetic diffusion. In the specific problem, we can neglect the magnetic field induction by comparing it with the applied magnetic field. Also, in the absence of the Hall force, the only effective force that remains is the electromagnetic force of Lorentz. The sequent assumptions are made: (i) the conducting fluids are Newtonian;(ii) properties of fluids are constants and appraised at the reference temperature; except the density, which varies linearly with temperature in the buoyancy term and is treated according to Boussinesq's approximation, (iii) Joule heating, and viscous dissipation terms are neglected in the following test. Similarly, (iv) the container walls are electrically insulated, and (v) radiation heat transfer is often ignored in the calculation.

\subsection{Governing Equations and Boundary Conditions}

The flow is axisymmetric $(\partial \partial \theta=0)$ where the model is expressed in cylindrical coordinates $(r, \theta, z)$. The governing magnetohydrodynamic mixed convection equations are dimensionless by the following quantities: the lengths $R$, the velocities $\Omega R$, the pressure $\rho(\Omega R)^{2}$, temperature, $\Theta=\left(T-T_{0}\right) / \Delta T$ and electric potential, $B_{0} \Omega R^{2}$. Also, it should be mentioned that only the steady flow is considered. So, the dimensionless equations can be defined as:

$$
\frac{1}{r} \frac{\partial\left(r v_{r}\right)}{\partial r}+\frac{\partial v_{z}}{\partial z}=0
$$


$\mathrm{v}_{r} \frac{\partial \mathrm{v}_{r}}{\partial r}-\frac{\mathrm{v}_{\theta}^{2}}{r}+\mathrm{v}_{z} \frac{\partial \mathrm{v}_{r}}{\partial z}=$

$-\frac{\partial \mathrm{p}}{\partial r}+\frac{1}{R e}\left(\frac{\partial^{2} \mathrm{v}_{r}}{\partial r^{2}}+\frac{1}{r} \frac{\partial \mathrm{v}_{r}}{\partial r}-\frac{\mathrm{v}_{r}}{r^{2}}+\frac{\partial^{2} \mathrm{v}_{r}}{\partial z^{2}}\right)+$

$\frac{H a^{2}}{R e} F_{L r}$

$\mathrm{v}_{r} \frac{\partial \mathrm{v}_{z}}{\partial r}+\mathrm{v}_{z} \frac{\partial \mathrm{v}_{z}}{\partial z}=$

$-\frac{\partial \mathrm{p}}{\partial z}+\frac{1}{R e}\left(\frac{\partial^{2} \mathrm{v}_{z}}{\partial r^{2}}+\frac{1}{r} \frac{\partial \mathrm{v}_{z}}{\partial r}+\frac{\partial^{2} \mathrm{v}_{z}}{\partial z^{2}}\right)+$

$\mathrm{Ri \Theta}+\frac{H a^{2}}{R e} F_{L z}$

$\mathrm{v}_{r} \frac{\partial \mathrm{v}_{\theta}}{\partial r}-\frac{\mathrm{v}_{r} \mathrm{v}_{\theta}}{r}+\mathrm{v}_{z} \frac{\partial \mathrm{v}_{\theta}}{\partial z}=$

$\frac{1}{\operatorname{Re}}\left(\frac{\partial^{2} \mathrm{v}_{\theta}}{\partial r^{2}}+\frac{1}{r} \frac{\partial \mathrm{v}_{\theta}}{\partial r}-\frac{\mathrm{v}_{\theta}}{r^{2}}+\frac{\partial^{2} \mathrm{v}_{\theta}}{\partial z^{2}}\right)+$

$\frac{H a^{2}}{R e} F_{L \theta}$

$\mathrm{v}_{r} \frac{\partial \Theta}{\partial r}+\mathrm{v}_{z} \frac{\partial \Theta}{\partial z}=\frac{1}{\operatorname{RePr}}\left(\frac{\partial^{2} \Theta}{\partial r^{2}}+\frac{1}{r} \frac{\partial \Theta}{\partial r}+\frac{\partial^{2} \Theta}{\partial z^{2}}\right)$

An electric current is induced by the interaction of convective flow with the magnetic field, $J=\sigma_{e}(\boldsymbol{E}+\boldsymbol{V} \times \boldsymbol{B})$ hence, the electric charges become as $\boldsymbol{E}=-\nabla \Phi$. Where $\mathbf{V}$ is the velocity vector $\left(\mathrm{v}_{r}, \mathrm{v}_{\theta}, \mathrm{v}_{z}\right): \mathrm{v}_{r}, \mathrm{v}_{\theta}$ and $\mathrm{v}_{z}$ for the radial, azimuthal and axial velocity components, respectively. The conservation of the induced electric current $\nabla \boldsymbol{J}=0$ gives the electric potential:

$\frac{1}{r} \frac{\partial}{\partial r}\left(r \frac{\partial \Phi}{\partial r}\right)+\frac{\partial^{2} \Phi}{\partial z^{2}}=\left(\frac{v_{\theta}}{r}+\frac{\partial v_{\theta}}{\partial r}\right)$

The Lorentz force is given by $\boldsymbol{F}_{L}=\boldsymbol{J} X \boldsymbol{B}$, which are in the $r, z$, and $\theta$ directions, respectively:

$$
\begin{gathered}
F_{L r}=-\mathrm{v}_{r} \\
F_{L z}=0 \\
F_{L \theta}=\frac{\partial \Phi}{\partial r}-\mathrm{v}_{\theta},
\end{gathered}
$$

and the dimensionless electric currents are:

$$
\left\{\begin{array}{c}
J_{r}=-\frac{\partial \Phi}{\partial r}+\mathrm{v}_{\theta} \\
J_{z}=-\frac{\partial \Phi}{\partial z} \\
J_{\theta}=-\mathrm{v}_{r}
\end{array}\right.
$$

Equation (4) for the swirling velocity is decoupled in this case from those of the other two velocity components. It is, therefore, possible to use the coupled equations for azimuthal velocity $\mathrm{v}_{\theta}$ and electric potential $\Phi$ (Eqs. (4) and (6)), to simplify the calculations and without solving the potential equation. For the present, the Hartmann number is smaller than 35 . So, it is possible to neglect the radial gradient of electric potential in comparison to azimuthal velocity $\left(\partial \Phi / \partial r \ll v_{\theta}\right)$, therefore Lorentz forces in azimuthal direction become $F_{L \theta} \approx-\mathrm{v}_{\theta}$ and $J_{r} \approx \mathrm{v}_{\theta}$, respectively. Here the Hartmann number $H a=B_{0} R \sqrt{\sigma_{e} /(v \rho)}$, is the ratio of electromagnetic force to the viscous force, the Reynolds number, $\operatorname{Re}=\Omega R^{2} / v$ is the rapport of inertial and viscous forces. The potential to kinetic energy rapport is defined as the Richardson number $R i=\beta g \Delta T / \Omega^{2} R$. Two other parameters control the behavior of the flow: the aspect ratio $H / R$ and the Prandtl number $\operatorname{Pr}=v / \alpha$. The symbols $\rho, v, \beta, \alpha$ and $\sigma_{e}$ denote, respectively, the density, the kinematic viscosity, the thermal expansion, the thermal diffusivity, and electric conductivity.

In all walls, no-slip boundary conditions are considered. The electrically insulated walls are defined by canceling the normal electric current density. Axi-symmetric boundary condition is specified along the axis $(\mathrm{r}=0)$, see Table 1 .

Table 1 Boundary conditions.

\begin{tabular}{|c|c|c|c|c|c|}
\hline Boundary & $\begin{array}{c}\text { Radial } \\
\text { velocity, }\end{array}$ & $\begin{array}{c}\text { Axial } \\
\text { velocity }\end{array}$ & $\begin{array}{c}\text { Azimuthal } \\
\text { velocity }\end{array}$ & Temperature & $\begin{array}{c}\text { Electric } \\
\text { potential }\end{array}$ \\
\hline $\mathrm{r}=0,0 \leq \mathrm{z} \leq \mathrm{H}$ & $\mathrm{v}_{r}=0$ & $\frac{\partial \mathrm{v}_{\mathrm{Z}}}{\partial r}=0$ & $\mathrm{v}_{\theta}=0$ & $\frac{\partial \Theta}{\partial r}=0$ & $\frac{\partial \Phi}{\partial r}=0$ \\
\hline $\mathrm{r}=1,0 \leq \mathrm{z} \leq \mathrm{H}$ & $\mathrm{v}_{r}=0$ & $\mathrm{v}_{z}=0$ & $\mathrm{v}_{\theta}=0$ & $\frac{\partial \Theta}{\partial r}=0$ & $\frac{\partial \Phi}{\partial r}=0$ \\
\hline $\mathrm{z}=0,0 \leq \mathrm{r} \leq 1$ & $\mathrm{v}_{r}=0$ & $\mathrm{v}_{z}=0$ & $\mathrm{v}_{\theta}=0$ & $\Theta=-1 / 2$ & $\frac{\partial \Phi}{\partial z}=0$ \\
\hline $\mathrm{z}=\mathrm{H} / \mathrm{R}, 0 \leq \mathrm{r} \leq 1$ & $\mathrm{v}_{r}=0$ & $\mathrm{v}_{z}=0$ & $\mathrm{v}_{\theta}=0$ & $\Theta=1 / 2$ & $\frac{\partial \Phi}{\partial z}=0$ \\
\hline
\end{tabular}




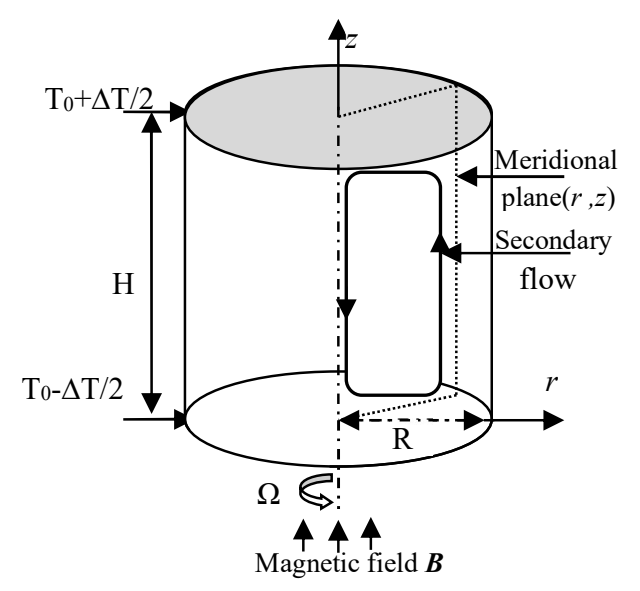

Fig.1. Flow geometry.

A streamfunction formulation is employed to implement the GITT approach (Lima et al. 2013; Matt et al. 2017; Quaresma et al. 2020). Before starting with the integral transformation of Eqs.(1)(5), the pressure field $P$ must be eliminated in the usual manner by defining the streamfunction $\Psi(r, z)$ as:

$\mathrm{v}_{r}=\frac{1}{r} \frac{\partial \Psi}{\partial z}, \mathrm{v}_{z}=-\frac{1}{r} \frac{\partial \Psi}{\partial r}$

Then, subtract the equations resulting from the differentiation of the Eqs. (2) and (3) to $\mathrm{z}$ and r, respectively. Both the continuity and the definition streamfunction equations are then used. Therefore, it results in the following system of three fourthorder and second-order partial differential equations and it can be written as:

$$
\begin{aligned}
& \frac{1}{r} \frac{\partial \Psi}{\partial z} \frac{\partial\left(\exists^{2} \Psi\right)}{\partial r}-\frac{1}{r} \frac{\partial \Psi}{\partial r} \frac{\partial\left(\exists^{2} \Psi\right)}{\partial z}- \\
& \frac{2}{r^{2}} \frac{\partial \Psi}{\partial z} \exists^{2} \Psi-2 \mathrm{v}_{\theta} \frac{\partial \mathrm{v}_{\theta}}{\partial z}= \\
& \frac{1}{R e} \exists^{4} \Psi-\operatorname{Rir} \frac{\partial \Theta}{\partial r}-\frac{H a^{2}}{\operatorname{Re}} \frac{\partial^{2} \Psi}{\partial z^{2}} \\
& \frac{1}{r} \frac{\partial \Psi}{\partial z}\left(\frac{\partial \mathrm{v}_{\theta}}{\partial r}+\frac{\mathrm{v}_{\theta}}{r}\right)-\frac{1}{r} \frac{\partial \Psi}{\partial r} \frac{\partial \mathrm{v}_{\theta}}{\partial z}= \\
& \frac{1}{\operatorname{Re}}\left(\frac{\partial^{2} \mathrm{v}_{\theta}}{\partial r^{2}}+\frac{1}{r} \frac{\partial \mathrm{v}_{\theta}}{\partial r}-\frac{\mathrm{v}_{\theta}}{r^{2}}+\frac{\partial^{2} \mathrm{v}_{\theta}}{\partial z^{2}}\right)-\frac{H a^{2}}{\operatorname{Re}}\left(\mathrm{v}_{\theta}\right) \\
& \frac{1}{r} \frac{\partial \Psi}{\partial z} \frac{\partial \Theta}{\partial r}-\frac{1}{r} \frac{\partial \Psi}{\partial r} \frac{\partial \Theta}{\partial z}=\frac{1}{\operatorname{RePr}} \nabla^{2} \Theta
\end{aligned}
$$

With associated dimensionless boundary conditions

$$
\begin{aligned}
& \lim _{r \rightarrow 0}\left[\frac{\Psi(r, z)}{r}\right]=0, \lim _{r \rightarrow 0}\left\{\frac{\partial}{\partial r}\left[\frac{1}{r} \frac{\partial \Psi(r, z)}{\partial r}\right]\right\}= \\
& 0, \mathrm{~V}_{\theta}(0, z)=0, \frac{\partial \Theta(0, z)}{\partial r}=0 \\
& \Psi(1, z)=\frac{\partial \Psi(1, z)}{\partial r}=\mathrm{v}_{\theta}(1, z)=\frac{\partial \Theta(1, z)}{\partial r}=0
\end{aligned}
$$

$$
\begin{aligned}
& \Psi(r, 0)=0, \frac{\partial \Psi(r, 0)}{\partial z}=0, \quad \mathrm{v}_{\theta}(r, 0)=r, \\
& \Theta(r, 0)=-\frac{1}{2} \\
& \Psi(r, H / R)=0, \frac{\partial \Psi(r, H / R)}{\partial z}=0, \\
& \mathrm{v}_{\theta}(r, H / R)=0, \Theta(r, H / R)=\frac{1}{2}
\end{aligned}
$$

Operators $\exists^{2}, \exists^{4}$ and $\nabla^{2}$ are defined as:

$\exists^{2}=\frac{\partial^{2}}{\partial r^{2}}-\frac{1}{r} \frac{\partial}{\partial r}+\frac{\partial^{2}}{\partial z^{2}}$

$\exists^{4}=\exists^{2}\left(\exists^{2}\right)=\frac{\partial^{4}}{\partial r^{4}}-\frac{2}{r} \frac{\partial^{3}}{\partial r^{3}}+\frac{3}{r^{2}} \frac{\partial^{2}}{\partial r^{2}}-$

$\frac{3}{r^{3}} \frac{\partial}{\partial r}-\frac{2}{r} \frac{\partial^{3}}{\partial r \partial z^{2}}+2 \frac{\partial^{4}}{\partial r^{2} \partial z^{2}}+\frac{\partial^{4}}{\partial z^{4}}$

$\nabla^{2}=\frac{\partial^{2}}{\partial r^{2}}+\frac{1}{r} \frac{\partial}{\partial r}+\frac{\partial^{2}}{\partial z^{2}}$

\subsection{Solution Methodology}

Three steps we need to follow in the GITT method for solving the MHD mixed convection problem. The first step is to provide the eigenvalues and eigenfunctions by choosing an appropriate eigenvalue problem. The definition of the integral transform pair is the second step and finally the integral transformation of Eqs. (9) - (11). The details of the GITT approach are given as follows, as an extension to hybrid solutions already detailed in reference (Quaresma et al. 2020 and Matt et al.2017). Hence, the eigenfunctions, eigenvalues, norms, and orthogonality properties for each potential in this problem determined, as follows:

- $\quad$ Streamfunction

$$
\begin{aligned}
& \frac{d^{4} X_{i}(r)}{d r^{4}}-\frac{2}{r} \frac{d^{3} X_{i}(r)}{d r^{3}}+ \\
& \frac{3}{r^{2}} \frac{d^{2} X_{i}(r)}{d r^{2}}-\frac{3}{r^{3}} \frac{d X_{i}(r)}{d r}= \\
& -\gamma_{i}^{2}\left[\frac{d^{2} X_{i}(r)}{d r^{2}}-\frac{1}{r} \frac{d X_{i}(r)}{d r}\right] \\
& \lim _{r \rightarrow 0}\left[\frac{X_{i}(r)}{r}\right]=0, \lim _{r \rightarrow 0}\left\{\frac{d}{d r}\left[\frac{1}{r} \frac{d X_{i}(r)}{d r}\right]\right\}=0 \\
& X_{i}(1)=0, \frac{d X_{i}(1)}{d r}=0
\end{aligned}
$$

The solution of the Eqs. (19)-(21) and the transcendental equation to calculate the eigenvalues $\gamma_{i}$ 's are determined, as follows:

$$
\begin{aligned}
& X_{i}(r)=r^{2}-\frac{r J_{1}\left(\gamma_{i} r\right)}{J_{1}\left(\gamma_{i}\right)}, \\
& J_{2}\left(\gamma_{i}\right)=0, \text { for } i=1,2,
\end{aligned}
$$


The orthogonality property of the eigenfunctions is expressed as:

$$
\begin{aligned}
& \int_{0}^{1} \frac{1}{r} \frac{d \mathrm{X}_{i}(r)}{d r} \frac{d \mathrm{X}_{j}(r)}{d r} d r= \begin{cases}0, & i \neq j \\
L_{i}, & i=j\end{cases} \\
& L_{i}=\int_{0}^{1} \frac{1}{r}\left[\frac{d \mathrm{X}_{i}(r)}{d r}\right]^{2} d r=\frac{\gamma_{i}^{2}}{2}
\end{aligned}
$$

- Azimuthal velocity

$\frac{1}{r} \frac{\mathrm{d}}{\mathrm{d} r}\left[r \frac{\mathrm{dY}_{i}(r)}{\mathrm{d} r}\right]-\left(\frac{1}{r^{2}}-\lambda_{i}^{2}\right) \mathrm{Y}_{i}(r)=0$

$\mathrm{Y}_{i}(0)=0, \mathrm{Y}_{i}(1)=0$

For azimuthal velocity the eigenquantities are expressed as:

$\mathrm{Y}_{i}(r)=J_{1}\left(\lambda_{i} r\right), J_{1}\left(\lambda_{i}\right)=0$, for $\mathrm{i}=1,2,3$,

$\int_{0}^{1} r Y_{i}(r) Y_{j}(r) d r= \begin{cases}0, & i \neq j \\ M_{i}, & i=j\end{cases}$

$M_{i}=\int_{0}^{1} r Y_{i}^{2}(r) d r=\frac{J_{0}^{2}\left(\lambda_{i}\right)}{2}$

- $\quad$ Temperature

$\frac{1}{r} \frac{d}{d r}\left[r \frac{d \Gamma_{i}(r)}{\mathrm{d} r}\right]+\mu_{i}^{2} \Gamma_{i}(r)=0$

$\frac{d \Gamma_{i}(0)}{d r}=0, \frac{d \Gamma_{i}(1)}{d r}=0$

For temperature the eigenquantities are expressed as:

$\Gamma_{i}(r)=J_{0}\left(\mu_{i} r\right), J_{1}\left(\mu_{i}\right)=0$, for $\mathrm{i}=1,2,3$,

$\int_{0}^{1} r \Gamma_{i}(r) \Gamma_{j}(r) d r= \begin{cases}0, & i \neq j \\ N_{i}, & i=j\end{cases}$

$N_{i}=\int_{0}^{1} r \Gamma_{i}^{2}(r) d r=\frac{J_{0}^{2}\left(\mu_{i}\right)}{2}$

The second step in the methodology comprises the definition of the integral transform pairs, which will permit the integral transformation of the partial differential equations in the streamfunction formulation of the problem. Thus, taking the following integral transform pairs for the potentials $(r, z), \mathrm{v}_{\theta}(r, z)$ and $\Theta(r, z)$, respectively, as:

$\bar{\Psi}_{i}(z)=-\frac{1}{L_{i}} \int_{0}^{1} \frac{d}{d r}\left[\frac{d \mathrm{X}_{i}(r)}{r d r}\right] \Psi(r, z) d r$ transform

$\Psi(r, z)=\sum_{i=1}^{\infty} X_{i}(r) \bar{\Psi}_{i}(z)$, inverse $\overline{\mathrm{v}}_{\theta, i}(\mathrm{z})=\frac{1}{\mathrm{M}_{i}} \int_{0}^{1} r Y_{i}(r) \mathrm{v}_{\theta}(r, z) d r$, transform

$\mathrm{v}_{\theta}(r, z)=\sum_{i=1}^{\infty} Y_{i}(r) \overline{\mathrm{v}}_{\theta, i}(z)$, inverse

$\bar{\Theta}_{i}(z)=\frac{1}{\mathrm{~N}_{i}} \int_{0}^{1} r \Gamma_{i}(r) \Theta(r, z) d r$, transform

$\Theta(r, z)=\sum_{i=1}^{\infty} \Gamma_{i}(r) \bar{\Theta}_{i}(z)$, inverse

The third step comprises the integral transformation of Eqs. (9) - (11) by multiplying these equations, together with the boundary conditions, by $X_{i}(r) / r$, $r Y_{i}(r)$ and $r \Gamma_{i}(r)$, respectively. Then, integrating over the domain $[0,1]$ in the radial direction and the inverse formulae are given by Eqs. (36), (38) and (40) are employed. After the usual manipulations, the following nonlinear coupled ordinary differential system (ODEs) is obtained for determining the transformed potentials $\bar{\Psi}_{i}(z), \overline{\mathrm{v}}_{\theta, i}(\mathrm{z}), \bar{\Theta}_{i}(z)$, respectively:

$$
\begin{aligned}
& \sum_{j=1}^{\infty} A_{i j} \frac{d^{4} \bar{\Psi}_{\mathrm{j}}(z)}{d z^{4}}=-\frac{\gamma_{i}^{4}}{2} \bar{\Psi}_{i}(z)+\gamma_{i}^{2} \frac{d^{2} \bar{\Psi}_{\mathrm{i}}(z)}{d z^{2}}+ \\
& H a^{2} \sum_{j=1}^{\infty} A_{i j}\left(\frac{d^{2} \bar{\Psi}_{\mathrm{i}}(z)}{d z^{2}}\right)+
\end{aligned}
$$

$\operatorname{Re}\left\{\begin{array}{l}\sum_{j=1}^{\infty} \sum_{k=1}^{\infty}\left[\begin{array}{l}\mathrm{B}_{\mathrm{ijk}} \bar{\Psi}_{\mathrm{j}}(z) \frac{d \bar{\Psi}_{\mathrm{k}}(z)}{d z}+ \\ \mathrm{C}_{\mathrm{ijk}} \frac{d \bar{\Psi}_{\mathrm{j}}(z) \frac{d^{2} \bar{\Psi}_{\mathrm{k}}(z)}{d z}+}{d z^{2}} \\ D_{i j k} \bar{\Psi}_{\mathrm{j}}(z) \frac{d^{3} \bar{\Psi}_{\mathrm{k}}(z)}{d z^{3}}\end{array}\right]+ \\ \sum_{j=1}^{\infty} \sum_{k=1}^{\infty} E_{i j k} \overline{\mathrm{v}}_{\theta, j}(z) \frac{d \overline{\mathrm{v}}_{\theta, \mathrm{k}}(z)}{d z}+R i \sum_{j=1}^{\infty} F_{i j} \bar{\Theta}_{j}(z)\end{array}\right\}$

$\frac{d^{2} \overline{\mathrm{v}}_{\theta, i}(z)}{\mathrm{dz}^{2}}=\lambda_{i}^{2} \overline{\mathrm{v}}_{\theta, i}(z)+H a^{2} \overline{\mathrm{v}}_{\theta, i}(z)+$

$\operatorname{Re} \sum_{j=1}^{\infty} \sum_{k=1}^{\infty}\left[\mathrm{G}_{\mathrm{ijk}} \overline{\mathrm{v}}_{\theta, \mathrm{j}}(z) \frac{d \bar{\Psi}_{\mathrm{k}}(z)}{d z}+\mathrm{H}_{\mathrm{ijk}} \frac{d \overline{\mathrm{v}}_{\theta, \mathrm{j}}(z)}{d z} \bar{\Psi}_{\mathrm{k}}(z)\right]$

$\frac{\mathrm{d}^{2} \bar{\Theta}_{i}(z)}{d z^{2}}=\mu_{i}^{2} \bar{\Theta}_{i}(z)+$

$\operatorname{RePr} \sum_{j=1}^{\infty} \sum_{k=1}^{\infty}\left[\begin{array}{c}I_{\mathrm{ijk}} \bar{\Theta}_{\mathrm{j}}(z) \frac{\mathrm{d} \bar{\Psi}_{\mathrm{k}}(z)}{\mathrm{dz}}+ \\ J_{\mathrm{ijk}} \frac{\mathrm{d} \bar{\Theta}_{j}(z)}{\mathrm{dz}} \bar{\Psi}_{\mathrm{k}}(z)\end{array}\right]$ 


$$
\begin{aligned}
& \bar{\Psi}_{i}(0)=0, \frac{d \bar{\Psi}_{i}(0)}{d z}=0, \\
& \overline{\mathrm{v}}_{\theta, i}(0) \bar{\Psi}_{i}(H / R)=\frac{d \bar{\Psi}_{i}(H / R)}{d z}= \\
& \overline{\mathrm{v}}_{\theta, i}(H / R)=0=-\bar{f}_{i}, \Theta_{i}(0)=-\frac{\overline{\mathrm{g}}_{i}}{2} \\
& \bar{\Psi}_{i}(H / R)=\frac{d \bar{\Psi}_{i}(H / R)}{d z}=\overline{\mathrm{v}}_{\theta, i}(H / R)=0 \\
& \bar{\Theta}_{i}(H / R)=\frac{\overline{\mathrm{g}}_{i}}{2}
\end{aligned}
$$

The integral coefficients are expressed as:

$$
\begin{aligned}
& A_{i j}=\int_{0}^{1} \frac{X_{i}(r) X_{j}(r)}{r} d r \\
& B_{i j k}=\int_{0}^{1} X_{i}(r)\left\{\begin{array}{l}
{\left[\begin{array}{l}
\frac{X_{j}^{\prime \prime}(r)}{r^{2}}-\frac{3 X_{j}^{\prime}(r)}{r^{3}}+\frac{3 X_{j}^{\prime}(r)}{r^{4}}
\end{array}\right]} \\
X_{k}(r)-\frac{X_{j}^{\prime}(r) X_{k}^{\prime \prime}(r)}{r^{2}}+ \\
\frac{X_{j}^{\prime}(r) X_{k}^{\prime}(r)}{r^{3}}
\end{array}\right\} d r \\
& C_{i j k}=\int_{0}^{1} X_{i}(r) X_{j}(r)\left[\frac{X_{k}^{,}(r)}{r^{2}}-\frac{2 X_{k}(r)}{r^{3}}\right] d r \\
& D_{i j k}=-\int_{0}^{1} \frac{X_{i}(r) X_{j}^{,}(r) X_{k}(r)}{r^{2}} d r \\
& E_{i j k}=-2 \int_{0}^{1} \frac{X_{i}(r) Y_{j}(r) Y_{k}(r)}{r} d r \\
& F_{i j}=\int_{0}^{1} X_{i}(r) \Gamma_{j}^{\prime}(r) d r \\
& G_{i j k}=\frac{1}{\mathrm{M}_{i}} \int_{0}^{1} Y_{i}(r)\left[Y_{j}(r)+\frac{Y_{j}(r)}{r}\right] X_{k}(r) d r \\
& H_{i j k}=-\frac{1}{\mathrm{M}_{i}} \int_{0}^{1} Y_{i}(r) Y_{j}(r) X_{k}(r) d r \\
& I_{i j k}=\frac{1}{\mathrm{~N}_{i}} \int_{0}^{1} \Gamma_{i}(r) \Gamma_{j}^{,}(r) X_{k}(r) d r, \\
& J_{i j k}=-\frac{1}{\mathrm{~N}_{i}} \int_{0}^{1} \Gamma_{i}(r) \Gamma_{j}(r) X_{k}^{,}(r) d r \\
& \bar{f}_{i}=\frac{1}{\mathrm{M}_{i}} \int_{0}^{1} r^{2} Y_{i}(r) d r, \quad \overline{\mathrm{g}}_{i}=\frac{1}{\mathrm{~N}_{i}} \int_{0}^{1} r \Gamma_{i}(r) d r
\end{aligned}
$$

The axial and radial velocities can be expressed in terms of the transformed streamfunction by using the definition of the streamfunction (Eq. (8)) and the inverse transform, as follows:

$$
\begin{aligned}
& \mathrm{v}_{r}(r, z)=\sum_{i=1}^{\infty} \frac{\mathrm{X}_{i}(r)}{r} \frac{\mathrm{d} \Psi_{i}(z)}{d z} \\
& \mathrm{v}_{z}(r, z)=-\sum_{i=1}^{\infty} \frac{1}{r} \frac{\mathrm{dX}_{i}(r)}{\mathrm{d} r} \bar{\Psi}_{i}(z)
\end{aligned}
$$

Also, via Eq. (2) the pressure field can be determined by substituting the relations for the three velocity components and then, performing an analytical integration in the radial direction. The electric current streamlines are traced using the electric streamfunction $\psi^{\prime}$ (Bojarevics et al.2009) defined as:

$$
J_{r}=1 / r . \partial \Psi^{\prime} / \partial z
$$

\section{NUMERICAL METHOD AND CONVERGENCE BEHAVIOR}

The coupled system of ordinary differential equations has been numerically solved with Fortran subroutine double-precision, namely DBVPFD from the IMSL Library (2018). The system of ordinary differential equations with associate boundary conditions can be solved by this subroutine at two points using a finite difference method (variable step-size) and variable order. The local relative error $10^{-4}$ is maintained automatically in the present study. It is known that the Roberts layers on the sidewall and Hartmann layers near to walls normal are influenced by increasing Hartmann number. Especially, the growth of $\mathrm{Ha}$ induces thinner Hartmann layers with $(\sim 1 / \mathrm{Ha})$ thickness. An attentive test of the convergence behavior of the results exposes that some terms in the expansions of $\mathrm{N}_{\psi}, \mathrm{N}_{\theta}$ and $\mathrm{N}_{\Theta}$ (Table 2) were adequate to guarantee at minimum three converged significant digits for the temperature and velocity field.

Table 2 Truncation orders of series.

\begin{tabular}{|l|c|c|c|}
\hline $\begin{array}{l}\text { Test } \\
\text { cases }\end{array}$ & $0<\mathrm{Ha} \leq 10$ & $10<\mathrm{Ha} \leq 20$ & $20<\mathrm{Ha} \leq 35$ \\
\hline $\begin{array}{l}\text { Case } \mathrm{A} \\
\mathrm{Re}=1500 \\
\mathrm{H} / \mathrm{R}=1.5\end{array}$ & $\mathrm{Nt}=100$ & $\mathrm{Nt}=110$ & $\mathrm{Nt}=120$ \\
\hline $\begin{array}{l}\mathrm{Case} \mathrm{B} \\
\mathrm{Re}=1855 \\
\mathrm{H} / \mathrm{R}=2.0\end{array}$ & $\mathrm{Nt}=150$ & $\mathrm{Nt}=165$ & $\mathrm{Nt}=180$ \\
\hline $\begin{array}{l}\mathrm{Case} \mathrm{C} \\
\mathrm{Re}=2400 \\
\mathrm{H} / \mathrm{R}=2.5\end{array}$ & $\mathrm{Nt}=200$ & $\mathrm{Nt}=220$ & $\mathrm{Nt}=240$ \\
\hline
\end{tabular}

The results reported in Table 3 for the streamfunction, temperature, and azimuthal velocity were computed for equal truncation orders $\mathrm{Nt}=\mathrm{N}_{\psi}=\mathrm{N}_{\theta}=\mathrm{N}_{\Theta}$. For three cases (A, B and C), the convergence of the GITT solution is analyzed for the values of the dimensionless streamfunction, azimuthal velocity, and temperature at the 
Table 3 Convergence of solution for the values of dimensionless streamfunction azimuthal velocity and temperature at monitoring point $(\mathrm{r}=0.5, \mathrm{z}=1.0)$

\begin{tabular}{|l|c|c|c|c|c|c|c|c|c|c|c|c|}
\hline \multicolumn{10}{|c|}{ Case $\mathrm{A}$ (Case B $\mathrm{Re}=1500, \mathrm{H} / \mathrm{R}=1.5)$} \\
\hline & \multicolumn{3}{|c|}{$\mathrm{Ha}=0$} & \multicolumn{3}{|c|}{$\mathrm{Ha}=15$} & \multicolumn{3}{c|}{$\mathrm{Ha}=25$} \\
\hline $\mathrm{Nt}$ & $\psi$ & $\Theta$ & $\mathrm{v}_{\theta}$ & $\mathrm{Nt}$ & $\psi$ & $\Theta$ & $\mathrm{v}_{\theta}$ & $\mathrm{Nt}$ & $\psi$ & $\Theta$ & $\mathrm{v}_{\theta}$ \\
\hline 80 & $3.26 \mathrm{e}-5$ & 0.1505 & 0.0301 & 90 & 0.00029 & 0.1588 & 0.1105 & 100 & 0.000381 & 0.1601 & 0.1580 \\
\hline 100 & $3.41 \mathrm{e}-5$ & 0.1576 & 0.0332 & 110 & 0.00032 & 0.1649 & 0.1161 & 120 & 0.000397 & 0.1698 & 0.1592 \\
\hline 120 & $3.41 \mathrm{e}-5$ & 0.1577 & 0.0335 & 130 & 0.00032 & 0.1649 & 0.1164 & 140 & 0.000397 & 0.1699 & 0.1592 \\
\hline
\end{tabular}

\begin{tabular}{|l|l|l|l|l|l|c|c|c|c|c|c|c|}
\hline \multicolumn{10}{|c|}{ Case B (Case B Re $=1855, \mathrm{H} / \mathrm{R}=2.0)$} \\
\hline & \multicolumn{3}{|c|}{$\mathrm{Ha}=0$} & & \multicolumn{3}{|c|}{$\mathrm{Ha}=15$} & \multicolumn{3}{c|}{$\mathrm{Ha}=25$} \\
\hline $\mathrm{Nt}$ & $\psi$ & $\Theta$ & $\mathrm{v}_{\theta}$ & $\mathrm{Nt}$ & $\psi$ & $\Theta$ & $\mathrm{v}_{\theta}$ & $\mathrm{Nt}$ & $\psi$ & $\Theta$ & $\mathrm{v}_{\theta}$ \\
\hline 140 & $1.76 \mathrm{e}-5$ & -0.0202 & 0.0601 & 155 & 0.00028 & -0.0144 & 0.1126 & 170 & 0.000329 & 0.0029 & 0.1594 \\
\hline 150 & $1.77 \mathrm{e}-5$ & -0.0206 & 0.0612 & 165 & 0.00029 & -0.0145 & 0.1131 & 180 & 0.000333 & 0.0031 & 0.1604 \\
\hline 160 & $1.77 \mathrm{e}-5$ & -0.0206 & 0.0613 & 175 & 0.00029 & -0.0145 & 0.1131 & 190 & 0.000333 & 0.0031 & 0.1605 \\
\hline
\end{tabular}

\begin{tabular}{|l|l|c|c|c|c|c|c|c|c|c|c|}
\hline \multicolumn{10}{|c|}{ Case C $(\mathrm{Re}=2400, \mathrm{H} / \mathrm{R}=2.5)$} \\
\hline & \multicolumn{3}{|c|}{$\mathrm{Ha}=0$} & \multicolumn{3}{|c|}{$\mathrm{Ha}=15$} & \multicolumn{3}{c|}{$\mathrm{Ha}=25$} \\
\hline $\mathrm{Nt}$ & $\psi$ & $\Theta$ & $\mathrm{v}_{\theta}$ & $\mathrm{Nt}$ & $\psi$ & $\Theta$ & $\mathrm{v}_{\theta}$ & $\mathrm{Nt}$ & $\psi$ & $\Theta$ & $\mathrm{v}_{\theta}$ \\
\hline 190 & $5.15 \mathrm{e}-6$ & -0.1151 & 0.0663 & 210 & 0.00026 & -0.1186 & 0.1167 & 230 & 0.000255 & -0.1092 & 0.1265 \\
\hline 200 & $5.32 \mathrm{e}-6$ & -0.1176 & 0.0686 & 220 & 0.00028 & -0.1198 & 0.1168 & 240 & 0.000260 & -0.1173 & 0.1280 \\
\hline 220 & $5.33 \mathrm{e}-6$ & -0.1177 & 0.0688 & 230 & 0.00029 & -0.1198 & 0.1169 & 250 & 0.000264 & -0.1172 & 0.1285 \\
\hline
\end{tabular}

monitoring point $(\mathrm{r}=0.5 ; \mathrm{z}=1.0)$. Table 3 reports the numerical results obtained when $R i=1.0$ and $P r$ $=0.65$, for increasing truncation order $\mathrm{Nt}$, respectively, for $H a=0, H a=15$ and $H a=25$, respectively. Truncation orders are increased up to 10 for the higher Hartmann number to attain convergence.

\section{RESUltS AND DISCUSSION}

First, these results obtained are validated with the results found in the literature. The reproduction of the hydrodynamic streamfunction $(\Psi)$ and electric one $\left(\Psi^{\prime}\right)$ for the case of $\mathrm{Re}=1750$ and $\mathrm{Ha}=5$ are shown in Fig.2. This reproduction is compared to the previous numerical model of Kharicha et al. (2004) who carried out a numerical and experimental combined study of steady laminar flow in a cylindrical cavity filled with mercury under a constant applied magnetic field
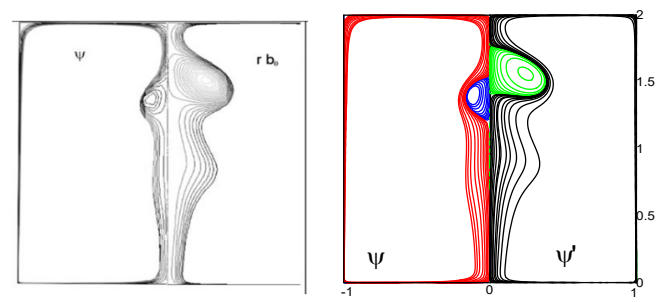

Fig. 2. Validation of the present results (right) against the numerical result of Kharicha et al. (2004) (left).

It is well known that the action of the axial magnetic field results in the stabilization of the flow (turbulent to laminar) or (asymmetric to axisymmetric). The cylinder is electrically insulated so that the electric current lines remain inside, which affects the movement and dampens the flow of the fluid especially the axial velocity. The Hartmann layers play the role of thermal insulation and favor the conduction, which in turn reduces the rate of heat transfer (Mahfoud and Bessaïh, 2016). The influence of a magnetic field on the vortex breakdown is well known from the recent of Laouari et al.(2021). However, the electromagnetic force reducing the pressure gradient and damping the axial movement of fluid, which tends to suppress the small vortex (counter-rotating flow). Magnetic field amplitude plays an important role when exceeds a certain value noted by the critical number of Hartmann ( $\left.\mathrm{Ha}_{\mathrm{cr}}\right)$, at this critical point the vortex breakdown disappears.

In this study, the computations were performed for three Prandtl numbers, $\operatorname{Pr}=\{0.025,0.032,0.065\}$, for Hartmann numbers $\mathrm{Ha}<35$. Three combinations of aspect ratios and Reynolds numbers (case A: $\mathrm{Re}=1500, \mathrm{H} / \mathrm{R}=1.5$ ); (case $\mathrm{B}: \mathrm{Re}=1855, \mathrm{H} / \mathrm{R}=2.0$ ) and (case $\mathrm{C}$ : $\mathrm{Re}=2400, \mathrm{H} / \mathrm{R}=2.5$ ) are compared. These cases corresponding to three forms of steadystate vortex breakdown. The Richardson number used in the simulation covers the range of $0 \leq \mathrm{Ri} \leq 2.0$. In the following sections, the magnetic field effects on the vortex breakdown and fluid layers are explored for each of these three cases.

\section{1 Magnetic Effect on Vortex Breakdown}

To investigate both position and suppression of the vortex hydrodynamic breakdown under a magnetic field we focus on the combination $(\mathrm{C}: \mathrm{Re}=2400$ and $\mathrm{H} / \mathrm{R}=2.5$ ) mentioned above. In $\mathrm{Ri}=0$, the forced convection is dominant and the flow structure is characterized by two bubbles merged to form a 'cucumber' bubble at $\mathrm{Ha}=1$. The hydrodynamic streamlines confirm that the cucumber bubble disappears at $\mathrm{Ha}=10$ and is given one-separationbubble (Fig. 4b). After that, the size of the hydrodynamic vortex drops slowly when the $\mathrm{Ha}$ is 


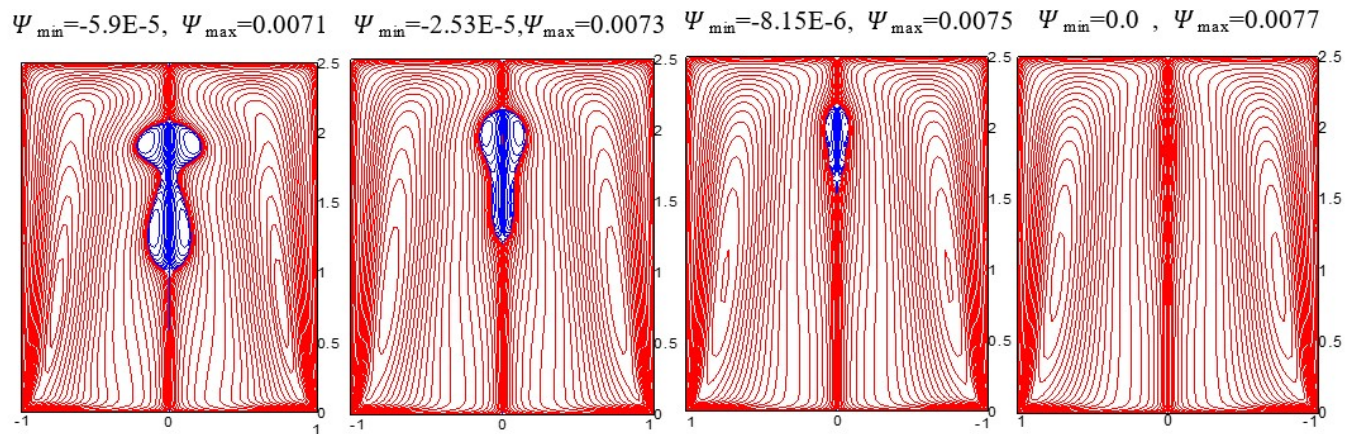

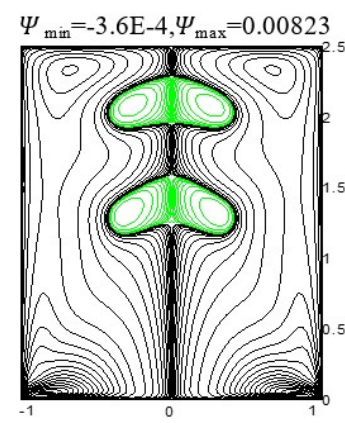

(a) $\mathrm{Ha}=1.0$

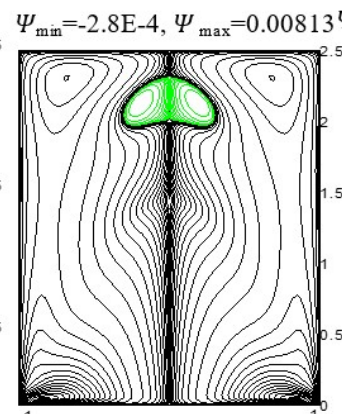

(b) $\mathrm{Ha}=10$

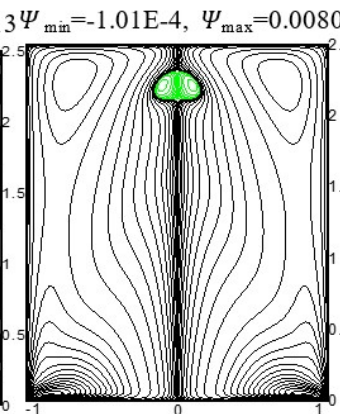

(c) $\mathrm{Ha}=15$

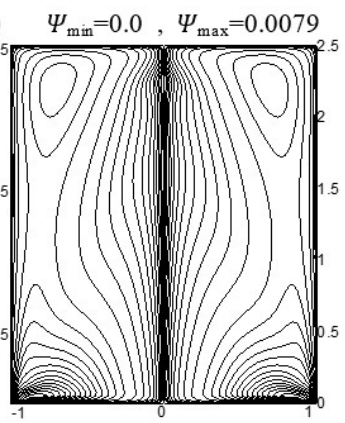

(d) $\mathrm{Ha}=18$

Fig. 3. Hydrodynamic streamlines $(\Psi)$ and electric current streamlines $\left(\Psi^{\prime}\right)$ with increasing Hartmann number at $R e=2400, R i=0$ and $H / R=2.5$.

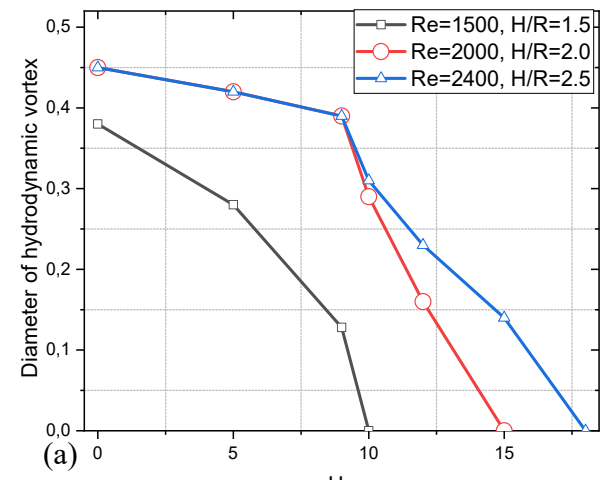

$\mathrm{Ha}$

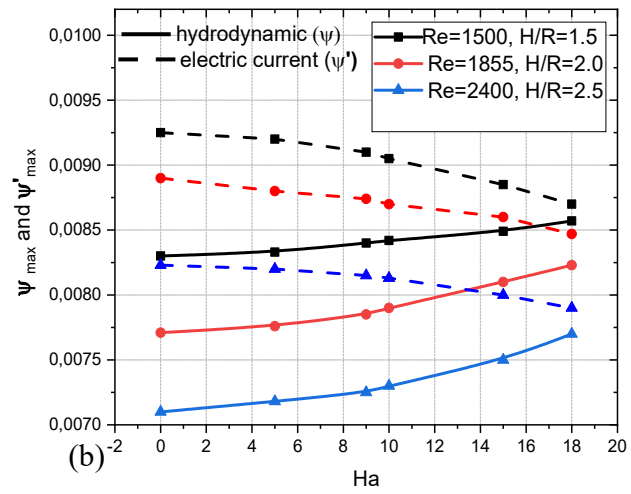

Fig. 4. Case of $R i=0$ at three cases (A, B and C): (a) hydrodynamic vortex diameter versus $\mathrm{Ha}$

(b) The hydrodynamic $(\Psi)$ and electric current $\left(\Psi^{\prime}\right)$ versus $\mathrm{Ha}$.

increased up to $\mathrm{Ha}=15$ and disappears at $\mathrm{Ha}_{\mathrm{cr}}=18$, see the first line of Fig. 4(d). The maximum stream function increase with increasing $\mathrm{Ha}$ and is 0.0077 at $\mathrm{Ha}_{\mathrm{cr}}=18$ and the minimum is zero value, which corresponds to the quasi-rigid rotation with an intermediate velocity. The dimensionless length of the vortex breakdown along the z-axis diminishes when $\mathrm{Ha}=1.0,10$, and 15 which is about $1.05,0.95$, and 0.58 , respectively. Finally, the hydrodynamic vortex disappears for the critical Hartmann number $\left(\mathrm{Ha}_{\mathrm{cr}}=18\right)$.

The second line of Fig. 3 shows the induced electric current lines, which show the existence of an electromagnetic vortex breakdown (Mahfoud et al. 2019). It seems that both hydrodynamic and electromagnetic vortices are connected because the disappearance of these two vortices is at the same critical Hartmann number (in this case $\mathrm{Ha}_{\mathrm{cr}}=18$ ). The origin of the creation of electric vortex is joint to the Lorentz forces in azimuthal direction $\left(F_{L \theta} \approx-\mathrm{v}_{\theta}\right)$ result from the vector product between the radial electric current, $J_{r}$, and the axial magnetic field, $B_{0} \mathbf{e}_{\mathrm{z}}$. For case $(\mathrm{C})$, the electric vortex appears immediately for $\mathrm{Ha}=1.0$, and the second separation bubble was found which is separated with a length 0.8 along the z-axis. There is only one electric vortex for $\mathrm{Ha}=10$ and its length along with the z-axis contracts in size at $\mathrm{Ha}=15$ and is about 0.3 . Finally, the electric vortex disappears when $\mathrm{Ha}_{\mathrm{cr}}=18$. The maximum of electric current streamlines diminishes with increasing $\mathrm{Ha}$ and is 0.00823 and 0.0079 for $\mathrm{Ha}=1.0$ and $\mathrm{Ha}=18$, respectively, contrary to the hydrodynamic streamlines where the maximum value increase with increasing Ha.

Figure 4a gives the evolution of the dimensionless diameters of the hydrodynamic vortex breakdowns 


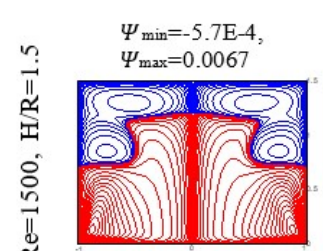

(a) $\mathrm{Ha}=0.0$

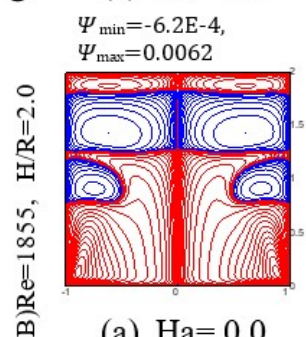

(a) $\mathrm{Ha}=0.0$

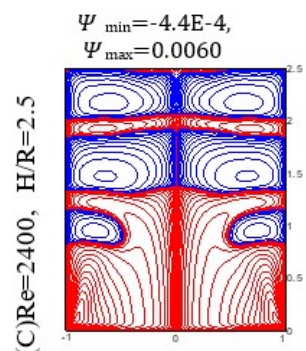

(a) $\mathrm{Ha}=0.0$

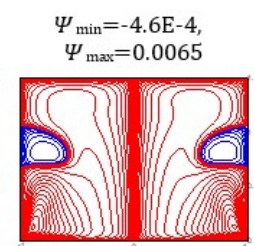

(b) $\mathrm{Ha}=10$

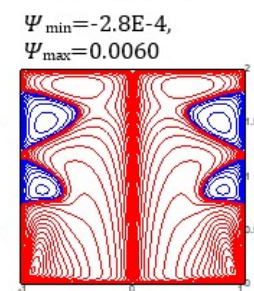

(b) $\mathrm{Ha}=10$

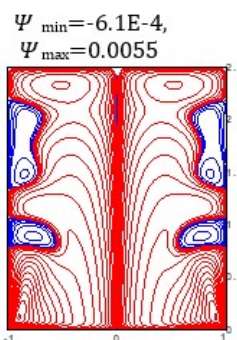

(b) $\mathrm{Ha}=10$

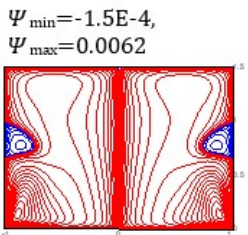

(c) $\mathrm{Ha}=15$

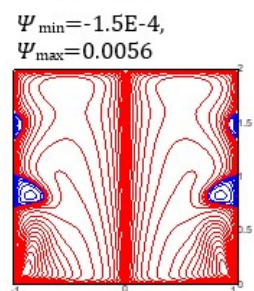

(c) $\mathrm{Ha}=15$

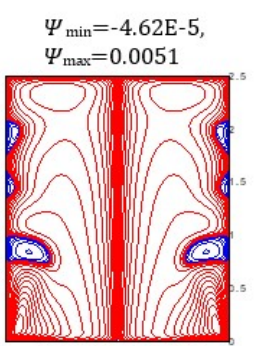

(c) $\mathrm{Ha}=15$

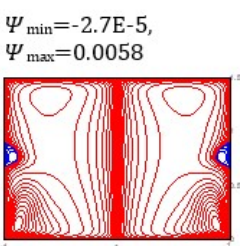

(d) $\mathrm{Ha}=20$

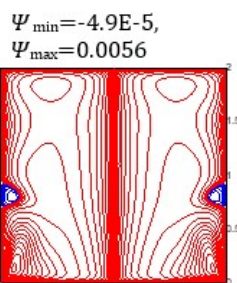

(d) $\mathrm{Ha}=20$

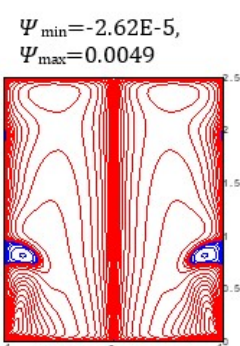

(d) $\mathrm{Ha}=20$

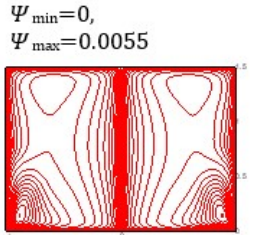

(e) $\mathrm{Ha}=24$

$\Psi_{\min }=0$

$\Psi_{\max }=0.0053$

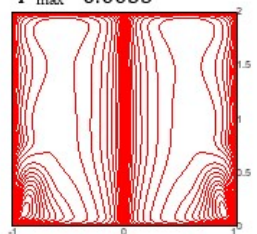

(e) $\mathrm{Ha}=26$

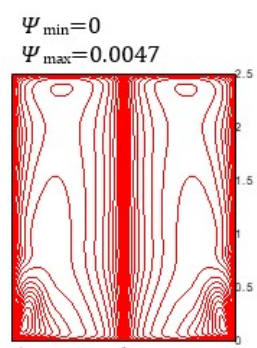

(e) $\mathrm{Ha}=30$

Fig. 5. Iso-contours of the stream function $\Psi$ when $\mathrm{Pr}=0.065$ and $\mathrm{Ri}=1$ for (a) $\mathrm{Ha}=0$, (b) $\mathrm{Ha}=10$, (c) $\mathrm{Ha}=15$, (d) $\mathrm{Ha}=20$, (e) $\mathrm{Ha}_{\mathrm{cr}}$.

as a function of the Hartmann number for all three cases (A, B, and $\mathrm{C})$. In case $\mathrm{A}$, the length of the diameter decrease with increasing $\mathrm{Ha}$ and is about 0.38 and 0.28 at $\mathrm{Ha}=0$ and 5, respectively. For $\mathrm{Ha}=$ 10 , the dimensionless diameter is zero and the vortex disappears completely. For cases, B and C the dimensionless diameters of the hydrodynamic vortex are gradually decreased with increasing $\mathrm{Ha}$ and record almost the same value for both cases up to $\mathrm{Ha}$ $=10$. For cases $\mathrm{B}$ and $\mathrm{C}$ the hydrodynamic vortex disappears when the Hartmann number is more than 15 and 18, respectively. Note that both the hydrodynamic and electric vortex breakdowns disappear at the same Hartmann number for all three cases mentioned above.

In Fig. $4 \mathrm{~b}$ the curves showing, the maximum hydrodynamic streamlines $(\Psi)$ and electric current streamlines $\left(\Psi^{\prime}\right)$ for three cases $(\mathrm{A}, \mathrm{B}$, and $\mathrm{C})$. The solid line presents the increasing of the maximum value of hydrodynamic streamlines $(\Psi)$ with increasing $\mathrm{Ha}$ for all three cases. On the contrary, the dashed lines represent the decreasing of the maximum value of electric streamlines $\left(\Psi^{\prime}\right)$ with increasing $\mathrm{Ha}$ for all three cases. This behavior proves that hydrodynamics and electric vortices seem to have an identical cause.

\subsection{Magnetic Effect on Fluid Layers}

To investigate the effects of the axial magnetic field on the layering (i.e., location or suppression), taking, for example, the case of $\mathrm{Pr}=0.065$ and $\mathrm{Ri}=1.0$ at three cases mentioned above (i.e. case A, B, and C, respectively). The hydrodynamic streamline plots in the case of $\mathrm{Ri}=1$ at $\mathrm{Ha}=0$ show a double layer, which the top layer, has a double lobe structure (plot in (A) of Fig. 5a). Some interesting changes are observed in the fluid layers when $\mathrm{Ha}$ is increased. As clearly shown by the streamlines on the first line of Fig. 5b, the clockwise recirculation top region diminishes in size and moves toward the sidewall when $\mathrm{Ha}=10$. On the contrary, the counterclockwise recirculation zone grows in size until it takes the entire top section of the cylinder. In addition, the maximum stream function decrease with increasing $\mathrm{Ha}$ and is 0.0067 and 0.0065 for $\mathrm{Ha}=0$ and $\mathrm{Ha}=10$, respectively. The small toroidal vortex decreases in size and then disappears at $\mathrm{Hacr}_{\mathrm{c}}=24$. The $\mathrm{r}$-central position of the small toroidal region rises with increasing $\mathrm{Ha}$ and, on the contrary, the z-central diminishes slightly. The central positions are at $\mathrm{z}=0.80,0.77$, and 0.76 for $\mathrm{Ha}$ $=10,15$ and 20, respectively. Similarly, on the r-axis are at $|\mathrm{r}|=0.78,0.83$, and 0.91 for $\mathrm{Ha}=10,15$, and 20, respectively (plot in (A) of Fig.5). When $\mathrm{Ha}=24$ at $\mathrm{Ri}=1$, the flow is characterized by a single layer in which the maximum streamfunction is $\Psi_{\max }=0.0055$.

As to case B shown in Fig 5, four layers occur at $H a=0$ in the same state (i.e, $P r=0.065$, and $\mathrm{Ri}=1$ ). The plots of streamline for $\mathrm{Ha}=10$, show only two layers. The length along the $\mathrm{z}$-axis of the clockwise toroidal vortex above $\mathrm{z}=1$ is become narrow and is about 0.47 . Another clockwise toroidal vortex exists 


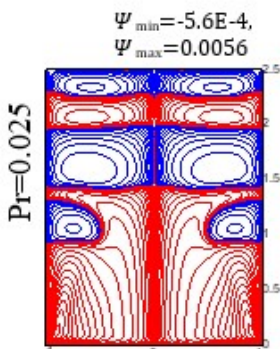

(a) $\mathrm{Ha}=0.0$

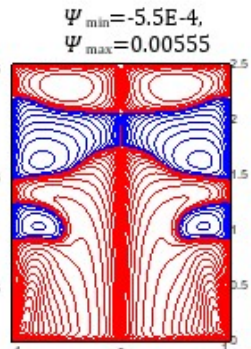

(b) $\mathrm{Ha}=5$

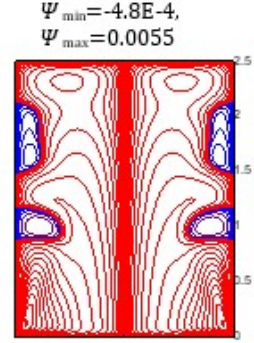

(c) $\mathrm{Ha}=10$

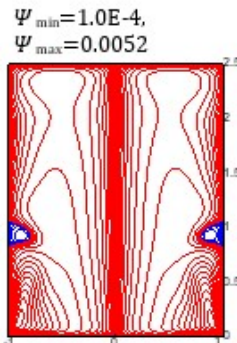

(d) $\mathrm{Ha}=20$

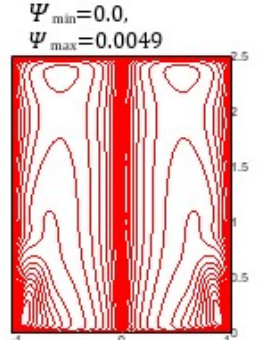

(e) $\mathrm{Ha}=26$

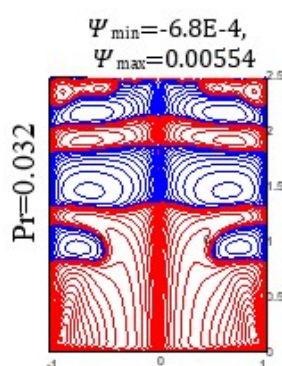

(a) $\mathrm{Ha}=0.0$

$\Psi_{\min }=9.8 \mathrm{E}-4$,

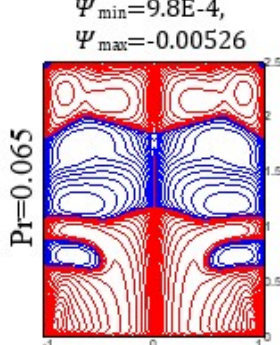

(a) $\mathrm{Ha}=0.0$

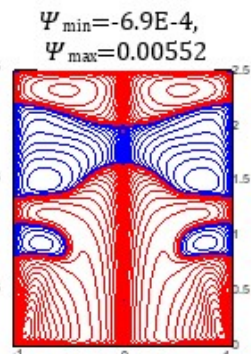

(b) $\mathrm{Ha}=5$

$\Psi_{\min }=9.2 \mathrm{E}-4$, $\Psi_{\max }=-0.00524$

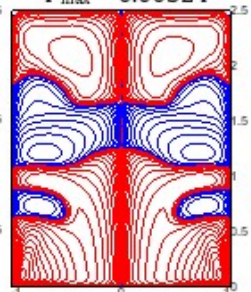

(b) $\mathrm{Ha}=5$

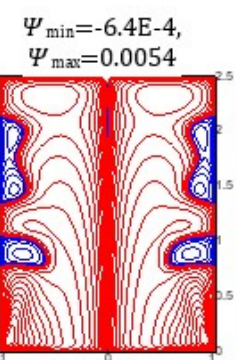

(c) $\mathrm{Ha}=10$

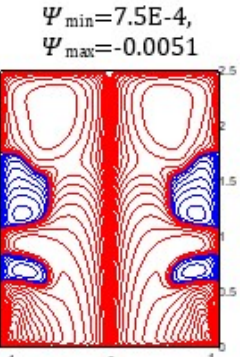

(c) $\mathrm{Ha}=10$

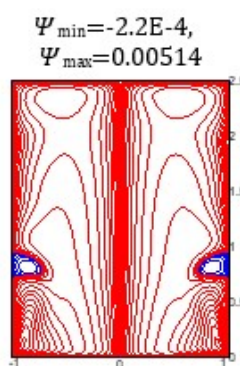

(d) $\mathrm{Ha}=20$

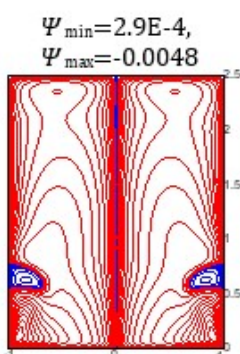

(d) $\mathrm{Ha}=20$

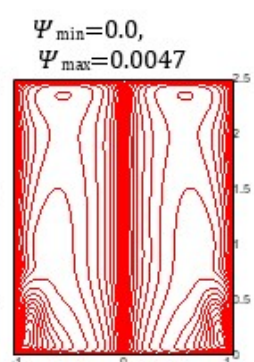

(e) $\mathrm{Ha}=28$

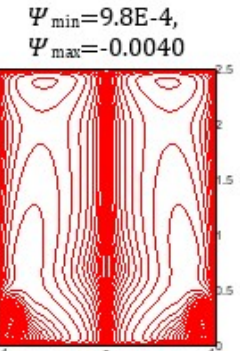

(e) $\mathrm{Ha}=35$

Fig. 6. Iso-contours of the stream function $\Psi$ for case $C(R e=2400, H / R=2.5)$ and $R i=2$ for $P r=0.025$ (top), $\mathrm{Pr}=0.032$ (middle), $\mathrm{Pr}=0.065$ (bottom): (a) $\mathrm{Ha}=0$, (b) $\mathrm{Ha}=10$, (c) $\mathrm{Ha}=15$, (d) $\mathrm{Ha}=20$, (e) $\mathrm{Ha}_{\mathrm{cr}}$.

below, which has a smaller size than the toroidal vortex that occurs above. The streamlines for $\mathrm{Ha}=15$ clearly show the small toroidal vortex above $\mathrm{z}=1$ decreases in size further and disappear at $\mathrm{Ha}=20$, but the vortex located under $\mathrm{z}=1$ always resists up to $\mathrm{Ha}=26$. Finally, the behavior of the flow is characterized by a single layer at $\mathrm{Ha}_{\mathrm{cr}}=26$ in which the maximum stream function decrease to 0.0053 .

The effect of increasing Ha is distinctly seen in Fig. 5 at case $\mathrm{C}$ with $\mathrm{Ri}=1$ and $\mathrm{Pr}=0.065$. For $\mathrm{Ha}=0$, six layers are observed, these layers begin to disappear as $\mathrm{Ha}$ rises to 10 and becomes only 3 layers. At $\mathrm{Ha}=15$ the clockwise toroidal vortex above $\mathrm{z}=1$ divides into two cells. The size of the toroidal vortex above $\mathrm{z}=1$ decreases with increasing $\mathrm{Ha}$ and disappear at $\mathrm{Ha}=22$, which gives a two-cell flow pattern. As clearly shown by the streamlines on the third line of Fig. 5e, the small toroidal vortex located under $\mathrm{z}=1$ decreases in size when $\mathrm{Ha}=25$ and disappears at $\mathrm{Ha}_{\mathrm{cr}}=30$. The $\mathrm{z}$-central location of this vortex rises with increasing Ha and, on the contrary, the absolute value of the r-central also decreasing.

For more details of the magnetic field effect on the suppression of fluid layers, taking the case $\mathrm{C}$ $(\mathrm{Re}=2400, \mathrm{H} / \mathrm{R}=2.5)$ and $\mathrm{Ri}=2$ for $\mathrm{Pr}=0.025$ (top),
$\operatorname{Pr}=0.032$ (middle), $\operatorname{Pr}=0.065$ (bottom) which is plotted in Fig.6. The streamline plots in the first line of Fig. 6 show the case of $\operatorname{Pr}=0.025$, which has five layers when $\mathrm{Ha}=0$. These layers merge and diminish in number which becomes four layers at $\mathrm{H}=5$, three at $\mathrm{Ha}=10$, and two layers at $\mathrm{Ha}=20$. Finally, only one layer stays when $\mathrm{Ha}_{\mathrm{cr}}=26$. Also, the maximum stream function decrease with increasing $\mathrm{Ha}$ and is 0.0056 and 0.0049 for $\mathrm{Ha}=0$ and $\mathrm{Ha}_{\mathrm{cr}}=26$, respectively. This behavior is in contrast to the isothermal cases $(\mathrm{Ri}=0)$ in which a rise in the maximum stream function can be observed because these layers seem to play the role of insulation.

Six layers are observed when $\mathrm{Pr}=0.032$ and $\mathrm{Ha}=0$ (plot in the second line of Fig. 6a). Some layers disappear with increasing Ha which has become four layers at $\mathrm{H}=5$, three at $\mathrm{Ha}=10$, and two layers at $\mathrm{Ha}=20$. The transition to a single layer structure occurs at the critical point $\mathrm{Ha}_{\mathrm{cr}}=28$. The maximum stream function decrease with increasing $\mathrm{Ha}$ and is 0.0047 when $\mathrm{Ha}_{\mathrm{cr}} 28$.

Contrary to the previous cases $(\operatorname{Pr}=0.025$ and $\mathrm{Pr}=0.026)$, it can be seen from the streamlines plotted in the third line of Fig. 6 that the number of fluid layers is decreased in the case of $\operatorname{Pr}=0.065$ which is 

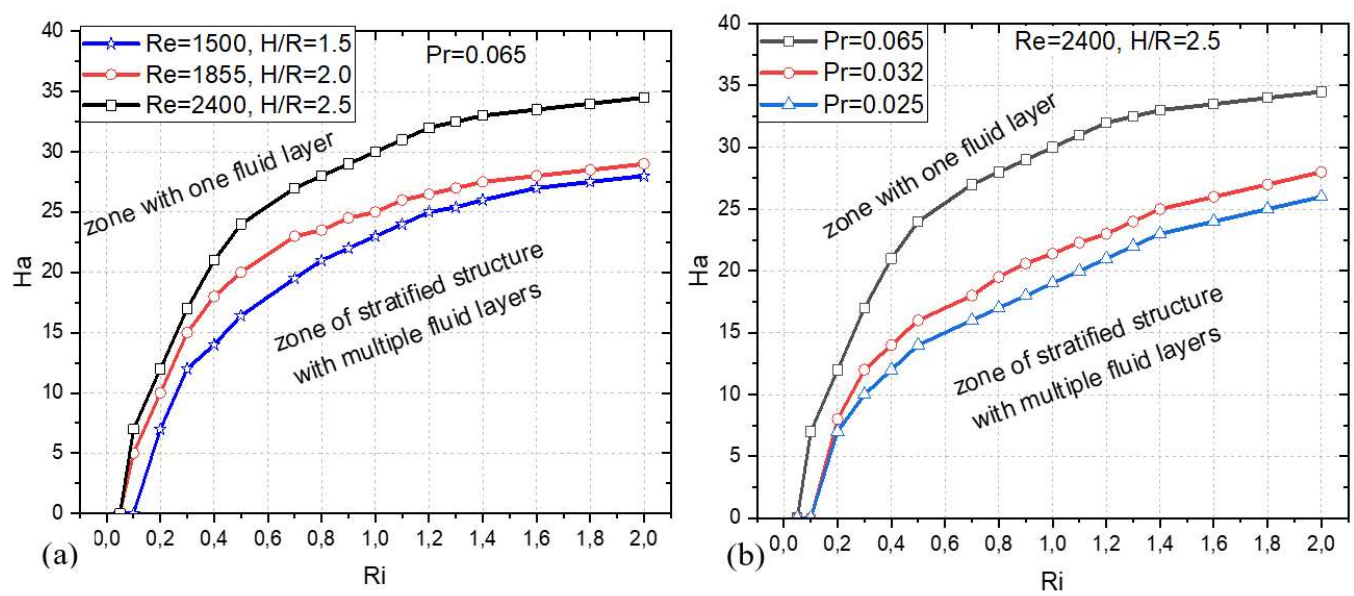

Fig.7. Evolution of $\mathrm{Ha}_{\mathrm{cr}}$ versus Ri for which shows the limits of the transition from the multiple fluid layers zone to the single-layer zone.

four layers when $\mathrm{Ha}=0$. The observation is almost the same as the previous cases in which four layers at $\mathrm{H}=5$, three at $\mathrm{Ha}=10$, and two layers at $\mathrm{Ha}=20$ are observed. For $\mathrm{Ha}_{\mathrm{cr}}=35$, the fluid which is characterized by a single layer rotates as a single block $\left(\Psi_{\min }=0\right) . \Psi_{\max }=0.004$ when $\mathrm{Ha}_{\mathrm{cr}}=35$ is the lowest value among the other critical values of the previous cases (i.e. $\operatorname{Pr}=0.025$ and $\operatorname{Pr}=0.032$ ).

The diagram in the $\left(\mathrm{Ha}_{\mathrm{cr}}-\mathrm{Ri}\right)$ plane for all three cases when $\mathrm{Pr}=0.065$ presented in Fig. $7 \mathrm{a}$ gives the evolution of critical Hartmann number $\mathrm{Ha}_{\mathrm{cr}}$, for which the stratified layers disappear versus the Reynolds number, Ri. There are three separate curves, represent the limits of two-zone, i.e., the domains with and without stratification fluid layers could be observed. The blue curve with stars symbols in Fig. 7a represents the boundary for multiple layers zone and the one layer zone for case A. The red curve with open circle symbols in Fig. 7a represents the critical Hartmann number for case B which the stratification fluid layers are suppressed and replaced by one layer. For case $\mathrm{C}$, the threshold of transition from the multiple layers zone to the one layer zone is plotted by the black curve with square symbols. In all cases, we have seen that the increase of the $\mathrm{Ri}$ causes the increase of the $\mathrm{Ha}_{\mathrm{cr}}$. Also, increasing $\mathrm{Ha}$ removes the fluid layers at a constant value of $\mathrm{Ri}$ (the layering disappears after the amplitude of Ha goes beyond a critical value). An exponential increase is indicated in the curves plotted in Fig. 7a. The magnitudes of $\mathrm{Ha}$ for case $\mathrm{C}$ are greater than those obtained in the cases of $\mathrm{A}$ and $\mathrm{B}$ for a fixed Richardson number. Taking $\mathrm{Ri}=1.0$ for example, the magnitudes of the critical Hartmann number are $\mathrm{Ha}=23,25$, and 30 for cases $\mathrm{A}, \mathrm{B}$, and $\mathrm{C}$, respectively.

Diagram, as shown in Fig. 7b, gives the evolution of transition from the multiple layers zone to the one layer zone for case A and Prandtl numbers given by $\operatorname{Pr}=0.025,0.032$, and 0.065 , respectively. The zone above the black curve with square symbols consisting of one layer appears at $\operatorname{Pr}=0.025$ for the values of the critical Hartmann number $\mathrm{Ha}_{\mathrm{cr}}=14,19$, and 26 correspondings respectively to the values of the $\mathrm{Ri}=0.5,1.0$, and 2.0. We have seen that the critical Hartmann number evolves with increasing $\mathrm{Ri}$. On the other hand, $\mathrm{Ha}_{\mathrm{cr}}$ rises with increasing Pr for a fixed value of Ri. Therefore, the values of $\mathrm{Ha}_{\mathrm{cr}}$ for case $\operatorname{Pr}=0.065$ are greater than those obtained in the cases of $\operatorname{Pr}=0.025$ and $\operatorname{Pr}=0.032$ ). It is observed that the divergence between the two curves $(\mathrm{Pr}=0.065$ and $\mathrm{Pr}=0.032)$ increases when $\mathrm{Ri}$ increases. By contrast, it can be seen from Fig. $7 b$ that the divergence between curves $\operatorname{Pr}=0.032$ and $\mathrm{Pr}=0.025$ is constant.

Figure 8a shows the influence of the magnetic field on the number of fluid layers and compares the three cases $(\mathrm{A}, \mathrm{B}$, and $\mathrm{C}$ ) mentioned above when $\mathrm{Pr}=0.065$ and $\mathrm{Ri}=1.0$.. The decrease in the curves as shown in Fig.8a indicates that increasing Ha has an important influence on the number of fluid layers formed. Therefore, the number of fluid layers decreases with increasing $\mathrm{Ha}$ for three cases. However, overall $\mathrm{Ha}$ has a non-monotonic effect on flow structure, because at $\mathrm{Ha}=15$ for case $\mathrm{C}$ it leading to a slight peak in the number of layers formed, here we have four layers. This result demonstrates that the axial magnetic field and thermal gradients can control the swirling flow and have the potential to reduce the stratification layers formed.

Figure $8 \mathrm{~b}$ compares the decreasing of the number of fluid layers with increasing $\mathrm{Ha}$ in case $\mathrm{C}$ for $\mathrm{Pr}=0.025,0.023$, and 0.065 respectively. For $\mathrm{Ha}=0$ we have six layers for $\operatorname{Pr}=0.032$, five layers for $\operatorname{Pr}=0.025$, and four layers for $\operatorname{Pr}=0065$. Over the range of $5 \leq \mathrm{Ha} \leq 25$, the decrease in the number of layers is the same for these three fluids. The flow is characterized by a single layer when $\mathrm{Ha}=26,28$ and 35 for $\operatorname{Pr}=0.025,0.032$ and 0.065 , respectively. Consequently, the Prandtl Number affects an increase or decrease in the number of resulting layers.

\section{CONCLUSION}

The effects of the magnetic field on laminar vortex breakdown and fluid layers development in steady- 

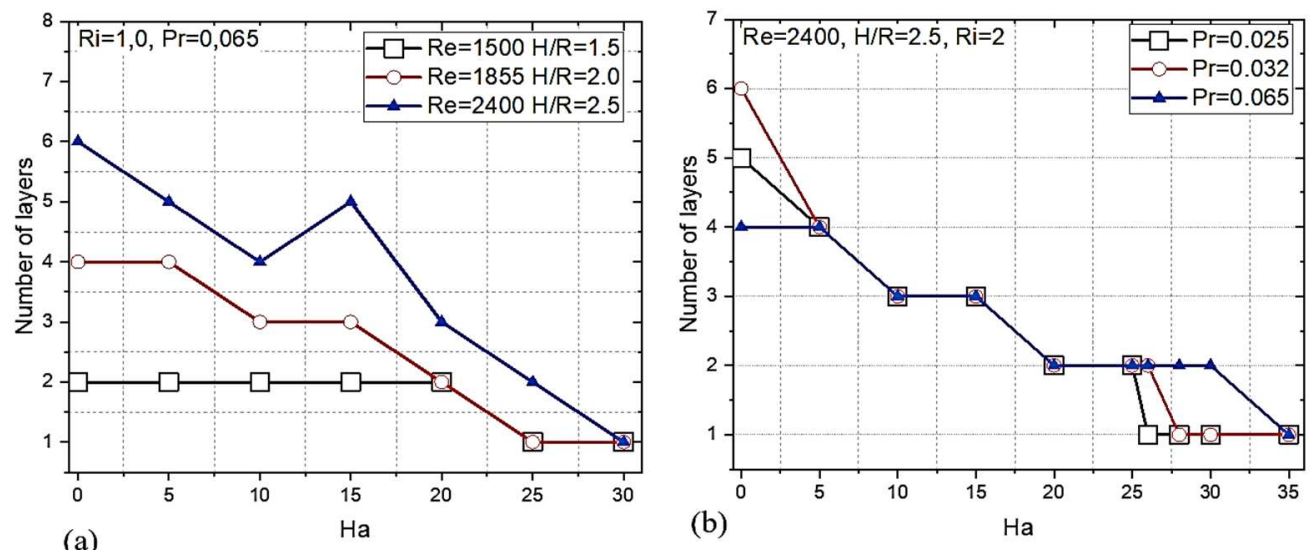

Fig. 8. Evolution of the number of layers VS Ha.

state mixed convection in cylindrical containers filled with a conducting viscous fluid with a bottom rotating disk have been numerically analyzed. The generalized integral transform technique (GITT) approach with a streamfunction formulation has been used to capture the different vortex breakdown in the isothermal case and the stratified layers under the temperature stratification condition. Three configurations of Reynolds numbers and aspect ratios and three Prandtl numbers were studied here. The main results obtained are as follows:

In the isothermal case, it was shown that the magnetic field can be used to control the position of both hydrodynamic and electric vortex breakdown and suppressed them at a critical value.

It looks that the suppression of both hydrodynamic and electric vortex is correlated with the same cause.

The Prandtl number and combination of (Reynolds number/aspect ratio) play an important role in the formation of fluid layers.

The results show that the increase of Ha causes a decrease in the number of fluid layers.

The present result shows that $\mathrm{Ha}_{\mathrm{cr}}$ (the transition from multiple fluid layers to one fluid layer) evolves with increasing Ri.

Finally, it should be mentioned that it is possible to solve the electric potential equation coupled with the momentum and energy equations.

\section{REFERENCES}

Bendjaghlouli, A., D. Ameziani, B. Mahfoud and L. Bouragbi (2019). Magnetohydrodynamic Counter Rotating Flow and Heat Transfer in a Truncated Conical Container. Journal of Thermophysics and Heat Transfer 33,865-74.

Bendjaghlouli, A., B. Mahfoud and D. E. Ameziani (2019).Magnetohydrodynamic flow in a truncated conical enclosure. Journal of Thermal Engineering 5, 77-83.
Bojarevics, V., J. A. Freibergs, E. I. Shilov and E. V. Shcherbinin (2009). Electrically Induced Vortical Flows. Published by Kluwer Academic Publishers, P.O. Box 17,3300 AA Dordrecht, The Netherlands;

Dash, S. C. and N. Singh (2019). Influence of Axial Magnetic Field on Swirling Flow and Vortex Breakdown Zones in a Cylindrical Cavity with a Rotating Lid. International Journal of Applied Mechanics 11, 1950054.

Davidson, P. A. (2001). An Introduction to Magnetohydrodynamics. Cambridge University Press, Cambridge.

IMSL ${ }^{\circledR}$ Fortran Numerical Library, Version (2018). Rogue Wave Software Inc., Boulder, USA, 2018.

Kharicha, A., A. Alemany and D. Bornas (2004) . Influence of the magnetic field and the conductance ratio on the mass transfer rotating lid driven flow. International Journal of Heat and Mass Transfer 47, 1997-2014.

Kharicha, A., A. Alemany and D. Bornas (2005) Hydrodynamic study of a rotating MHD flow in a cylindrical cavity by ultrasound shift method. International Journal of Engineering Science 43(7), 589-615.

Laouari, A., B. Mahfoud, R. Bessaïh and A. Hadjadj (2021). Hydrodynamic instabilities in swirling flow under axial magnetic field. European Journal of Mechanics-B/Fluids 85, 245-260.

Lima, J. A. and M. G. O. Rêgo (2013). On the integral transform solution of low-magnetic MHD flow and heat transfer in the entrance region of a channel. International Journal of Non-Linear Mechanics 50, 25-39.

Mahfoud, B., A. Bendjaghlouli and R. Bessaïh (2016). Magneto-hydrodynamic co-rotating flow in a vertical cylindrical container. Numerical Heat Transfer, Part A, 69 (9),1051106.

Mahfoud, B., A. Laouari, A. Hadjadj and $\mathrm{H}$. Benhacine (2019). Counter-rotating flow in 
B. Mahfoud / JAFM, Vol. 14, No. 6, pp. 1741-1753, 2021.

coaxial cylinders under an axial magnetic field. European Journal of Mechanics-B/Fluids, 78,139-46.

Mahfoud, B. and R. Bessaïh (2012). Stability of swirling flows with heat transfer in a cylindrical enclosure with co/counter-rotating end disks under an axial magnetic field. Numerical Heat Transfer, Part A, 61(6),463-482.

Mahfoud, B. and R. Bessaïh (2016). Magnetohydrodynamic counter-rotating flow in a cylindrical cavity. International Journal of Heat and Mass Transfer 93,175-185.

Mahfoud, B., H. Benhacine, A. Laouari and A. Bendjaghlouli (2020). Magnetohydrodynamic Effect on Flow Structures Between Coaxial Cylinders Heated from Below. $J$. Thermophysics and Heat Transfer 34, 265-274.
Matt, C. F., J. N. Quaresma and R. M. Cotta (2017). Analysis of magnetohydrodynamic natural convection in closed cavities through integral transforms. International Journal of Heat and Mass Transfer 113, 502-513.

Oreper, G. and J. Szekely (1984) . The effect of a magnetic field on transport phenomena in a Bridgman-Stockbarger crystal growth. Journal of Crystal Growth 6(7),405-19.

Quaresma, N.N., C. da Cruz, N. Cagney, R. M. Cotta and S. Balabani (2020). Effect of mixed convection on laminar vortex breakdown in a cylindrical enclosure with a rotating bottom plate. International Journal of Thermal Science 155, 106399.

Yu, Y., B-W. Li and A. Thess (2013). The effect of a uniform magnetic field on vortex breakdown in a cylinder with rotating upper lid. Computers \& Fluids 88, 510-23. 